\title{
Synthesis and Characterization of Gold Nanoparticles with Plasmon Absorbance Wavelength Tunable from Visible to Near Infrared Region
}

\author{
Weihong Shi, ${ }^{1,2}$ Justin Casas, ${ }^{1}$ Meenakshi Venkataramasubramani, ${ }^{1}$ and Liang Tang ${ }^{1}$ \\ ${ }^{1}$ Department of Biomedical Engineering, University of Texas at San Antonio, San Antonio, TX 78249, USA \\ ${ }^{2}$ Department of Medical Technology, Yancheng Health Vocational and Technical College, Jiangsu, Yancheng 224006, China
}

Correspondence should be addressed to Liang Tang, liang.tang@utsa.edu

Received 31 May 2012; Accepted 26 June 2012

Academic Editors: G. Dzhardimalieva, G.-H. Kim, and G. Skordaris

Copyright () 2012 Weihong Shi et al. This is an open access article distributed under the Creative Commons Attribution License, which permits unrestricted use, distribution, and reproduction in any medium, provided the original work is properly cited.

Gold nanorods with localized surface plasmon resonance (LSPR) can be chemically synthesized. We systematically investigated the effects of reaction parameters and centrifugation on the fine tuning of the rod dimension in scale-up production ( $80-100 \mathrm{~mL}$ ). Nanorods of absorption bands from $600-1050 \mathrm{~nm}$ were fabricated with precise control of the aspect ratio (AR) from 1.5 to 8.9. Although all chemicals are important in directing the nanostructure, silver ion concentration and seed/Au ${ }^{3+} \mathrm{ratio}$ were the most effective variations to adjust the absorption wavelength. With a single surfactant under the influence of silver nitrate, short nanorods up to AR of 5 were synthesized with corresponding maximum absorption wavelength at $902 \mathrm{~nm}$. To achieve higher aspect ratio with absorption band beyond $1,000 \mathrm{~nm}$, two-surfactant growth solution was sought to further elongate the rod length. Centrifugation speed and times were found to exert significant influences on the final rod dimension, which is important during the purification process. In a relatively large quantity nanorod synthesis, even distribution and sufficient mixing of chemical ingredients play an essential role in determining the yield, uniformity, and stability of the final nanorod formation.

\section{Introduction}

Nanoparticles provide unique physical and optical properties to serve as building blocks for a wide-ranging applications in nanoelectronics, nanophotonics, nanodevices, and nanomedicine [1-5]. Particularly, nanoscale gold exhibits properties which are fundamentally different from other nanoparticles [6-8]. The small size comparable to biological molecules, intense photophysical properties, and high efficiency of heat conversion from light absorption have made gold nanoparticle a huge popular nanomaterial for biomedical diagnostic (e.g., bioanalysis, nanophotonics) and therapeutic (e.g., drug delivery, hyperthermia) applications.

Gold nanoparticles of varying morphologies and sizes can be fabricated by photolithography techniques and biosynthetic organisms. Nevertheless, chemical synthesis may be one of the favored and cost-effective routes. Nowadays, nanomaterials of various shapes can be precisely synthesized with seemingly limitless chemical functional groups.
A variety of gold nanoparticles including nanospheres, nanorods, nanocages, nanoshells, nanoprisms, nanocubes, and nanorings have been chemically fabricated with high yield [7-12]. Among these geometries, nanorod is particularly interesting to us, because it provides an excellent platform with well-defined absorption spectrum for a labelfree bioanalytical assay [13-18]. The optical transduction by $\mathrm{Au}$ nanorods (GNRs) is based upon the phenomenon of localized surface plasmon resonance, that is, nanoSPR (or LSPR) [19-21]. The longitudinal band is a stronger band corresponding to electron oscillation along the long axis of the nanorod. The optical absorption is very sensitive to dielectric change in the vicinity of nanorods. Upon biological binding, for example, can cause a detectable shift in the peak wavelength of absorption spectrum. The magnitude of the shift is directly proportional to the concentration of bound proteins.

Numerous characteristics of nanoparticles depend on aspect ratios (length to width ratio), including optical and 
physical properties. As such, the longitudinal absorption wavelength can be selectively tuned from the visible to the near-infrared (NIR) region as aspect ratio (length to width ratio) increases. This provides a unique opportunity to develop a multiplexed nanodiagnostic device based on combination of different size of nanorods with distinctive plasmonic wavelengths (ongoing project). Therefore, control over the shape and size of fabricated nanorods precisely has been one of the important tasks to provide the desired nanomaterials for targeted biomedical applications. Most of the past studies discussed the influence of the various reaction parameters in the seed-mediated rod growth in a relatively small quantity $(5-10 \mathrm{~mL})$. In a scale-up production, precise tunability of the plasmon bands from visible to NIR region has not been thoroughly studied. This is important as the mass transport, therdynamics, and mixing effect can be dramatically different between small quantify and large volume. Here, we performed a systematic study to investigate the effects of various parameters in an $80-100 \mathrm{~mL}$ production volume. Additionally, centrifugation is an essential step to purify rod-shaped nanoparticles from excess chemicals and spheres. We found that centrifuge can significantly affect the final dimension and optical absorption of prepared GNR samples. Therefore, we systematically investigated, for the first time, the influence of centrifuge speed and times on the preparation, properties, and stability of nanorods.

\section{Materials and Methods}

2.1. Materials. Hydrogen tetrachloroaurate trihydrate $\left(\mathrm{HAuCl}_{4} ; 99 \%\right)$, sodium borohydride $\left(\mathrm{NaBH}_{4} ; 99 \%\right)$, cetyltrimethylammonium bromide (CTAB), L-ascorbic acid (AA), silver nitrate $\left(\mathrm{AgNO}_{3} ; 99 \%\right)$, and benzyldimethylhexadecylammonium chloride (BDAC) were obtained from Sigma-Aldrich (St. Louis, MO). All glasswares were cleaned with aqua regia $\left(\mathrm{HCl} / \mathrm{HNO}_{3}, 3: 1 \mathrm{v} / \mathrm{v}\right)$, followed by thorough rinse with double distilled MilliQ water prior to use.

2.2. Fabrication of $A u$ Nanorods. Gold nanorods with various aspect ratios up to 9 demonstrating tunable longitudinal plasmonic wavelengths $(600-1050 \mathrm{~nm})$ were synthesized as described previously with modifications [22-24].

2.2.1. Nanorods with Plasmon Peak $<900 \mathrm{~nm}$. Typically, $\mathrm{HAuCl}_{4}(5 \mathrm{~mL}, 0.5 \mathrm{mM})$ was mixed with CTAB $(5 \mathrm{~mL}, 0.2 \mathrm{M})$ solution, followed by addition of freshly made, ice cold $\mathrm{NaBH}_{4}(600 \mu \mathrm{L}, 10 \mathrm{mM})$ under rapid mixing for $2 \mathrm{~min}$. $\mathrm{NaBH}_{4}$ is a strong reducing agent which resulted in a brownish-yellow color. The mixture was then allowed to stand without disturbance at $25-27^{\circ} \mathrm{C}$ for at least 2 hours before use as aseed solution. To fabricate Au nanorods, the seeds were then added to a growth solution and incubated at $27^{\circ} \mathrm{C}$ overnight to allow complete rod growth. The growth solution was typically prepared as follows. To make an $80 \mathrm{~mL}$ of rod solutions, $40 \mathrm{~mL} \mathrm{HAuCl} 4(1 \mathrm{mM})$ was first mixed with $40 \mathrm{~mL}$ CTAB solution $(0.2 \mathrm{M})$. Depending on the desired rod dimension and longitudinal plasmonic wavelength, appropriate amount of $\mathrm{AgNO}_{3}(4 \mathrm{mM})$ was added to the mixture, followed by $560 \mu \mathrm{L}$ AA solution $(78.8 \mathrm{mM})$. The mixture turned colorless instantly after rapid mixing. The rod growth process was initiated after adding $96 \mu \mathrm{L}$ seed solution as prepared above. The color of the mixture would usually change within minutes, suggesting an engaged growth of seed particles. This method typically produces short nanorods with absorption peak up to $900 \mathrm{~nm}$.

2.2.2. Nanorods with Plasmon Peak $>900 \mathrm{~nm}$. A binary surfactant mixture of BDAC/CTAB is essential in the seedmediated growth solution to fabricate longer nanorods with higher aspect ratio $>5$ and plasmonic band over $1,000 \mathrm{~nm}$. Briefly, the two-surfactant mixture was prepared by adding $0.8 \mathrm{~g}$ of CTAB to $0.15 \mathrm{M}$ BDAC solution. After dissolving the mixture by sonication $\left(20 \mathrm{~min}\right.$ at $\left.40^{\circ} \mathrm{C}\right)$, the solution was added to $200 \mu \mathrm{L}$ of $4 \mathrm{mM} \mathrm{AgNO}_{3}$, followed by addition of $5 \mathrm{~mL}, 1 \mathrm{mM} \mathrm{HAuCl}_{4}$. Adding $70 \mu \mathrm{L}$ of $78 \mathrm{mM}$ ascorbic acid and lastly $12 \mu \mathrm{L}$ Au seed solution initiated the rod growth. Instead of overnight incubation, the process continued over a period of 25 days with close monitoring of the absorption spectrum profile. The solution was periodically sampled to assess the temporal evolution of the long nanorods.

2.3. Purification of Au Nanorods. The synthesized Au nanorods were purified to remove excess reagents such as CTAB surfactant by centrifugation. Typically, the solution was centrifuged at a speed of $8,500 \mathrm{rpm}$ for $30 \mathrm{~min}$ to precipitate the Au nanorod solid. The colorless supernatant was carefully discarded without disturbing the bottom. The solid pellet was then redispersed in a suitable volume of DI water depending on the quantity of the residue. The centrifugation was repeated 2-3 times, and finally the solid residue was dispersed in DI water or biological buffers for biofunctionalization process.

2.4. Characterization. Absorption spectra of the prepared $\mathrm{Au}$ nanorod solutions were obtained on a Beckman-Coulter UVNIR (200-1100 nm) scan spectrophotometer. The SEM grid was dropcast with a total of $10 \mu \mathrm{L}$ of the purified rod solution and allowed to air-dry in the open atmosphere. Electron microscopy images of the nanorods were taken using Hitachi scanning electron microscope (SEM), and elemental analysis was carried out on a Joel SEM equipped with X-ray energy dispersive analysis (EDAX). For each sample, the size of 200 particles was measured to obtain the average rod dimension, aspect ratio, and yield. The yield was calculated by number of rods divided by total number of particles $(\times 100 \%)$.

\section{Results and Discussion}

Nanorods are formed based on the structure-directing elongation of seeds. The seeds are basically $5-10 \mathrm{~nm}$ spheres which demonstrate a characteristic absorption band of gold at $520 \mathrm{~nm}$. In contrast, rod-shaped nanoparticles have two bands in the absorption spectrum (Figure 1(a)). The lower peak around $520 \mathrm{~nm}$ represents the surface plasmon resonance along transverse direction similar to that from nanospheres. The second peak which is dominant arises from 


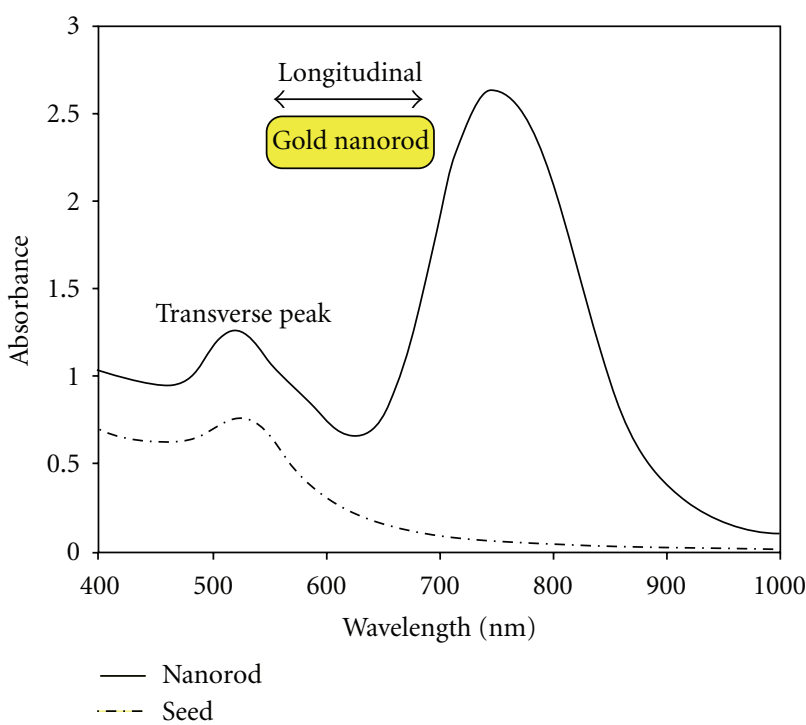

(a)

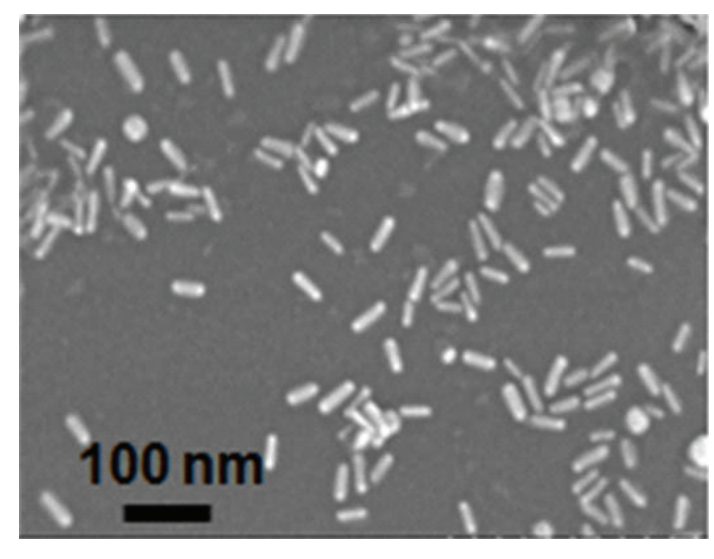

(b)

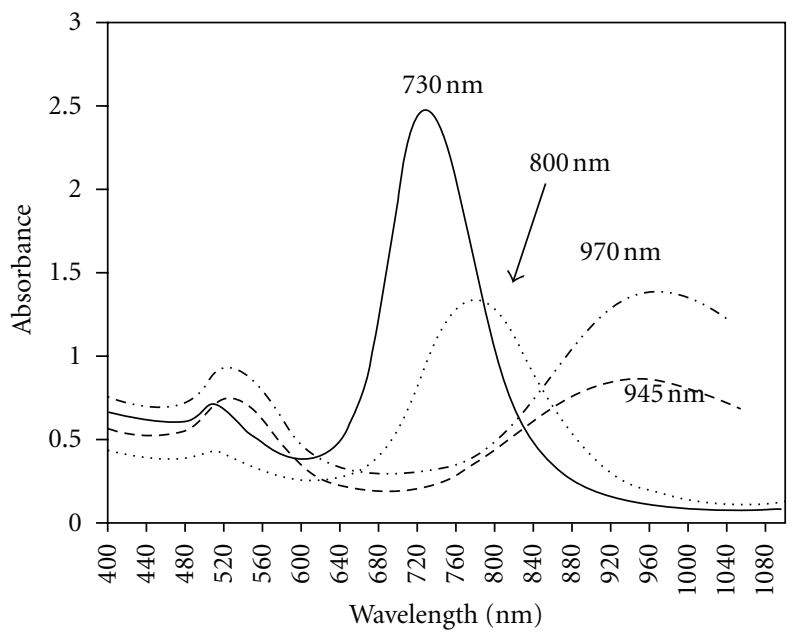

(d)

FIGURE 1: (a) Representative absorption spectrum of gold nanorod with stronger band in the longitudinal direction. Gold seed (nanosphere) only shows a characteristic band (dashed line). (b) Scanning electron microscopy of rod-shaped gold nanoparticles. (c) Picture demonstrating a vivid color of the gold nanorod colloidals with tunable aspect ratio (AR) to result in distinguishable absorption wavelength from 650 to $1050 \mathrm{~nm}$. The solutions are stable over 6 months. (d) Superimposed absorption spectrum of Au nanorods with various longitudinal peak wavelengths.

the surface plasmon along the longitudinal direction. As observed in SEM pictures, the rod dimensions are usually 50 $100 \mathrm{~nm}( \pm 5-20 \mathrm{~nm})$ in length and $10-40 \mathrm{~nm}( \pm 3-10 \mathrm{~nm})$ in width. Depending on the nanoparticle aspect ratio (length to width ratio), the peak wavelength can be selectively tuned from visible to NIR region. As the aspect ratio increases from 1.5 to 8.9 , the longitudinal peak wavelength shifts from 650 to $1050 \mathrm{~nm}$. Unlike bulk or molecular scale, nanoscale gold nanorods exhibit a variety of vivid colors. The colloidal solutions demonstrate distinguishable colors from blue to brown to light pink and red, which correspond to the respective light absorption wavelengths (Figure 1(c)). The suspension solution is stable over six months at room temperature. Figure $1(\mathrm{~d})$ shows the representative vis-NIR absorption spectrum of nanorods with different plasmonic wavelengths. Regardless of the dimension, the lower peaks from transverse are always around $510-540 \mathrm{~nm}$ which is characteristic surface plasmon of gold. Only the dominant longitudinal peaks shift in visible and NIR region as the nanoparticles gradually grow longer in the length under influence of chemicals in the growth solution. Although all the ingredients are essential for the rod growth, we found that the silver nitrate amount and seed/Au ${ }^{3+}$ ratio are the most effective players in precise control of rod shape and size. In the following sections, we describe optimization of each parameter individually in a $80-100 \mathrm{~mL}$ production volume. 


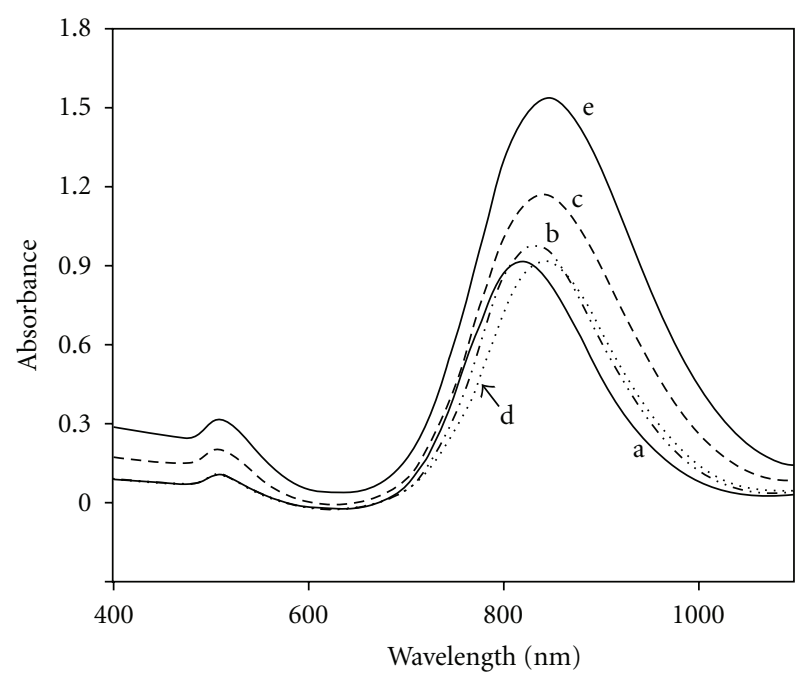

(a)

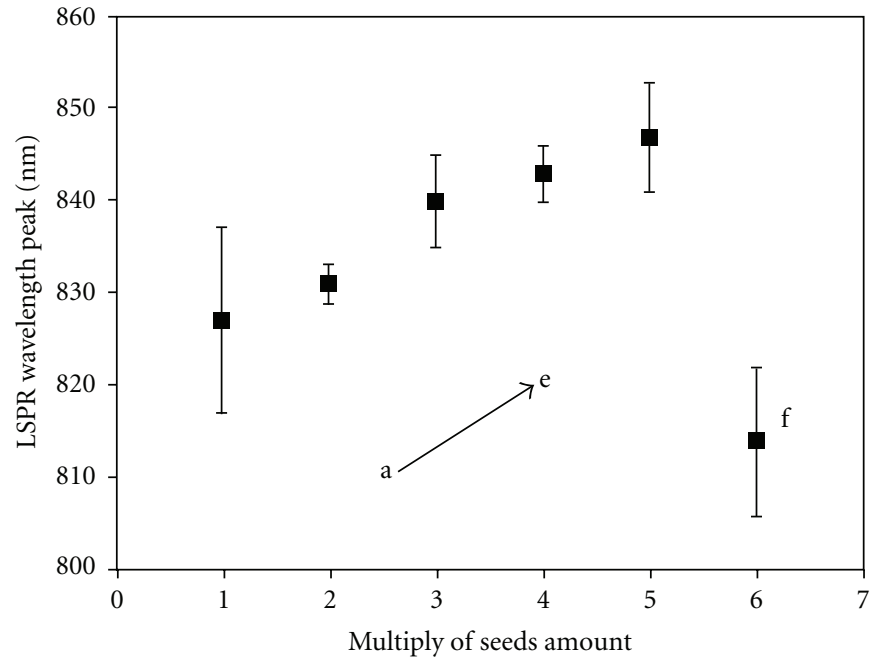

(b)

FIGURE 2: Effect of seed amount on the nanorod growth. (a) One to five times (a to e) of the normal seed amount (12 $\mu \mathrm{L}$ ) cause a shift of the longitudinal plasmon to a longer wavelength. (b) Excess amount of seed $>5$ times (f) results in a reduction in the aspect ratio and therefore a shorter SPR wavelength.

We also discuss the influence of centrifuge on the nanorod purification, shape, size, and stability as it is an essential process to prepare GNR sample for further applications.

3.1. Effect of Seed Amount. Gold nanosphere $(5-10 \mathrm{~nm})$ is required in the growth solution to serve as a material basis (seed) to allow attachment of gold atoms in a controlled direction to grow to a rod shape under influence of surfactant and silver nitrate. While the concentrations of other ingredients were kept constant, the amount of seeds added to the growth media was varied with increasing volume up to 6 times of normal amount $(12 \mu \mathrm{L})$. SEM examination found that the rod length decreased with an increase in the seed amount, which was expected due to the increased competition among seed particles for a given concentration of $\mathrm{Au}^{3+}$ ion. In other words, there is a lesser quantity of $\mathrm{Au}^{3+}$ ions available for growth per seed particle. Despite the reduction in the length, addition of more seed solution (curves a to e: 1-5 times of seed solution) caused a red shift in the longitudinal plasmon peak wavelength (Figure 2(a)). This observation is sort of contradicting with the decrease in the rod length. However, the simultaneous reduction in rod width altogether actually leads to an increasing aspect ratio [22]. It is known that larger aspect ratio correlates with longer wavelength in the absorption spectrum. It should be noted that addition of excessive seed amount $>5$ times would cause a decrease in the aspect ratio and result in a shorter plasmonic peak wavelength. Six times of seed concentration cause a reduction of the absorption peak from 850 to $810 \mathrm{~nm}$ (Figure 2(b)). This is probably due to depletion of the $\mathrm{Au}^{3+}$ ions over excessive seeds and ultimately very short rods with a small number of spheres appeared.
3.2. Effect of Ascorbic Acid Amount. During the rod growth, gold (III) ions will be reduced to atomic elements for material supply to Au seeds to grow favorably in length under the influence of silver nitrate. When faster supply of monomer growth unit $\left(\mathrm{Au}^{0}\right)$ is available to the seeds, there is a great tendency to induce the growth of seed particles in all directions and forming more spherical particles. AA is a mild reducing agent and a suitable amount of AA will facilitate the synthesis of nanorods with high yield. We found that in our experimental condition, $0.1 \mathrm{M}$ AA was an optimal concentration to produce rods in high yield. Quantity less than that stoichiometric amount required for the reduction of $\mathrm{Au}^{3+}$ ions to $\mathrm{Au}^{0}$ failed to grow rods. For example, colloidal solution with $0.08 \mathrm{M}$ AA did not produce a characteristic absorption peak of rod shape (Figure 3(a)). Excess of AA, however, usually resulted in a "dogbone" shape of rods (Figure 3(b)). Depending on the faceting of the ends of the rods, additional plasmon bands can be observed [25]. Our study corroborated this finding. In addition to the usual transverse and longitudinal plasmon absorbance peaks, one additional medium peak was observed in the absorption spectrum when $0.18 \mathrm{M}$ AA was added. The mechanism of the production of "dogbone" shape is under investigation.

3.3. Effect of Silver Nitrate Amount. Silver ion is known to play an important role in assisting the growth of nanoparticles to rod shape. It is especially essential for the synthesis of short nanorods $(\mathrm{AR}<5)$ with peak wavelength in visible region of the spectrum in very high yield $(>95 \%)$ [22]. In our study, we found that varying the silver ion amount is also the most effective way to fabricate rods with tunable LSPR wavelength from $600-900 \mathrm{~nm}$ in large quantity. Little silver nitrate $(<50 \mu \mathrm{L}, 4 \mathrm{mM})$ often resulted 


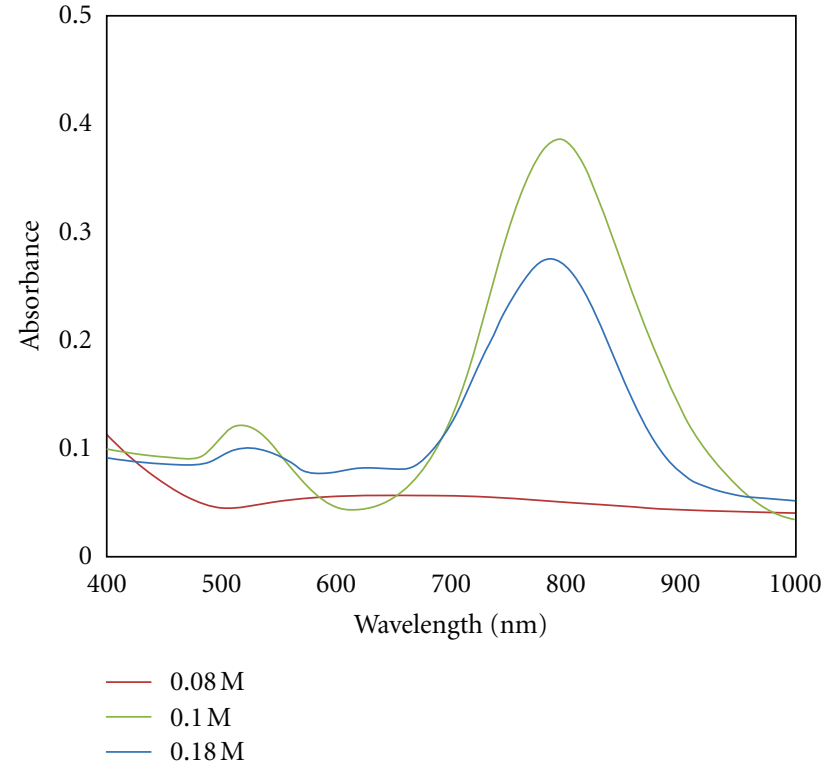

(a)

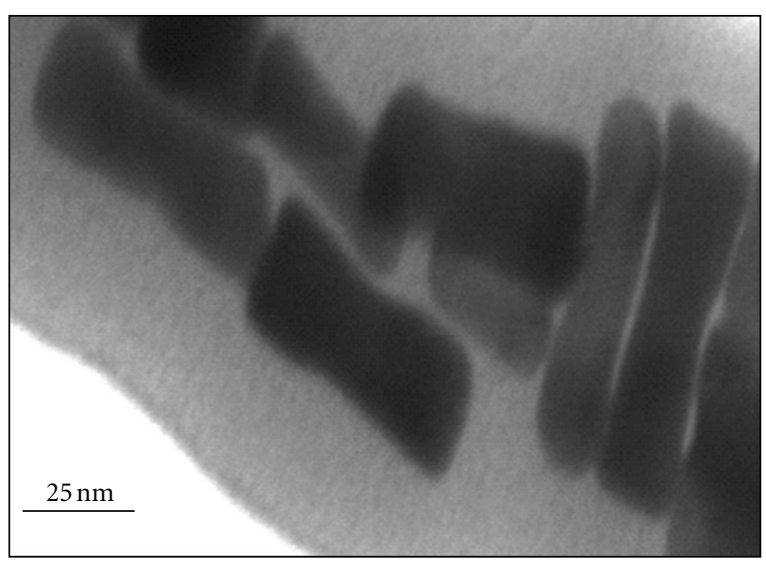

(b)

FIgURE 3: Optimization of ascorbic acid (AA) amount for nanorod growth. Addition of excess AA causes "dogbone" shaped rod.

in few rod-shaped nanoparticles. Instead, only nanospheres were confirmed from single peak around $520 \mathrm{~nm}$ in the absorption spectrum, indicating the essential role of silver ions in rod growth. As the addition of silver nitrate amount increased, the length of the rod increased and most importantly the aspect ratio increased (Figure 4). The longitudinal peak wavelength can be fine-tuned from 600 to $902 \mathrm{~nm}$ depending on the volume of the added $\mathrm{AgNO}_{3}$ $(4 \mathrm{mM})$. This is probably because more $\mathrm{Au}^{0}$ is zipped into the rod-shaped soft template assisted by more available silver ions along the longitudinal direction. However, the effect of the silver ion concentration is not always to increase the aspect ratio of rods thereby resulting in a longer absorption wavelength. When increasing the amount of the $\mathrm{AgNO}_{3}$ $(4 \mathrm{mM})$ in the growth solution to more than $350 \mu \mathrm{L}$, the peak wavelength became shorter, indicating a decrease in the

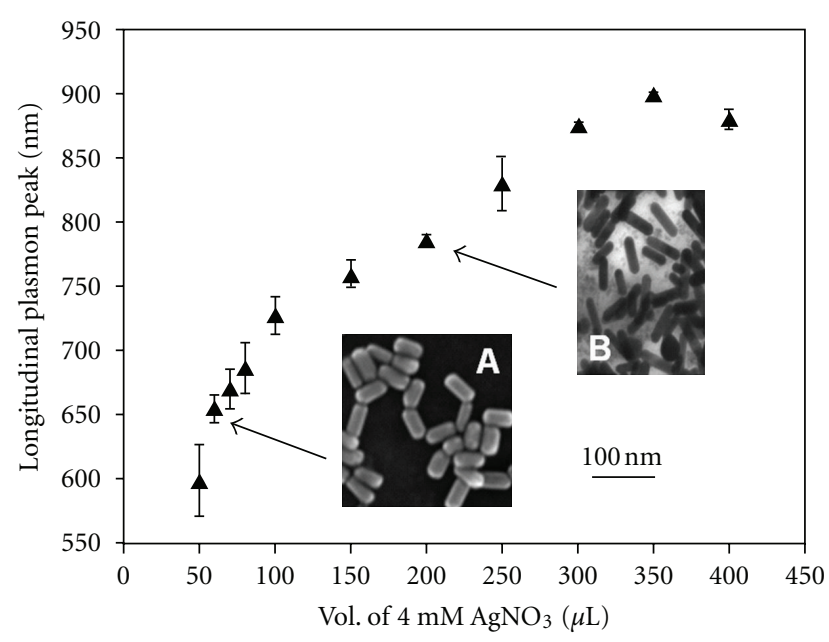

FIgURE 4: Additional amount of silver nitrate can effectively increase the aspect ratio and fabricate nanorods with a maximum absorption wavelength at $902 \mathrm{~nm}$ using a single CTAB surfactant. A: LSPR wavelength: $655 \pm 11 \mathrm{~nm}$; Aspect ratio: $2.5 \pm 0.2$. B: $787 \pm 4 \mathrm{~nm}$; aspect ratio: $4.1 \pm 0.3$.

aspect ratio of the rods. The decrease in the size at the high concentration of silver ions could be due to the ionic strength effect and/or depletion of $\mathrm{Au}^{3+}$ in the growth solution [24].

Although the mechanism to assist in the rod shape growth is not clearly elucidated, several studies $[22,24]$ suggested that $\mathrm{AgNO}_{3}$ forms $\mathrm{AgBr}$ in the presence of CTAB, and $\mathrm{AgBr}$ adsorbs differentially to the facets of gold particles thereby restricting their growth to the rod shape and stabilizing the nanorod surface. This speculation is consistent with the explanation that silver nitrate and CTAB synergistically function as a soft template to favor elongation of the seed to rod shape. Our EDAX data from electron microscope also confirmed the presence of bromine $(2.65 \pm 0.1 \% \mathrm{wt})$ coming from $\mathrm{CTAB}$ on the nanorod surfaces. Moreover, as mentioned above, when faster supply of monomer growth unit $\left(\mathrm{Au}^{0}\right)$ is available to the seeds, there is a great tendency to induce the growth of seed particles in all directions and form more spherical particles. This was evidenced by our study results of little rod production without addition of silver nitrate. However, in the presence of silver nitrate, the kinetics of reduction of gold ions to atomic gold is slower. This fact may play an essential role in the high yield of rodshaped nanoparticles under the $\mathrm{CTAB}-\mathrm{AgNO}_{3}$ system.

\subsection{Growth of Au Nanorods with Plasmon Peak Wavelength >} $900 \mathrm{~nm}$. Au nanorods with longer absorption wavelength in NIR region are beneficial for many biological applications such as thermal therapy and imaging $[6,7]$. The surfactantassisting growth of nanorods described above only uses a single surfactant, that is, CTAB. Although the longitudinal SPR wavelength can be effectively tuned by increasing the amounts of $\mathrm{AgNO}_{3}$ and/or seed amounts, this method generally fabricates rods of aspect ratio up to 5 with absorption at $<900 \mathrm{~nm}$. Excessive addition of the silver ions 


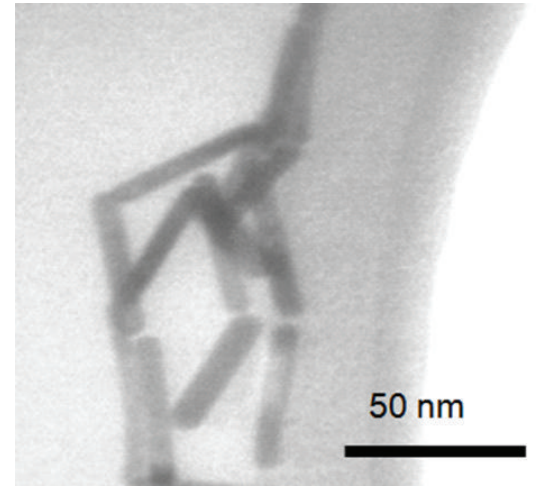

(a)

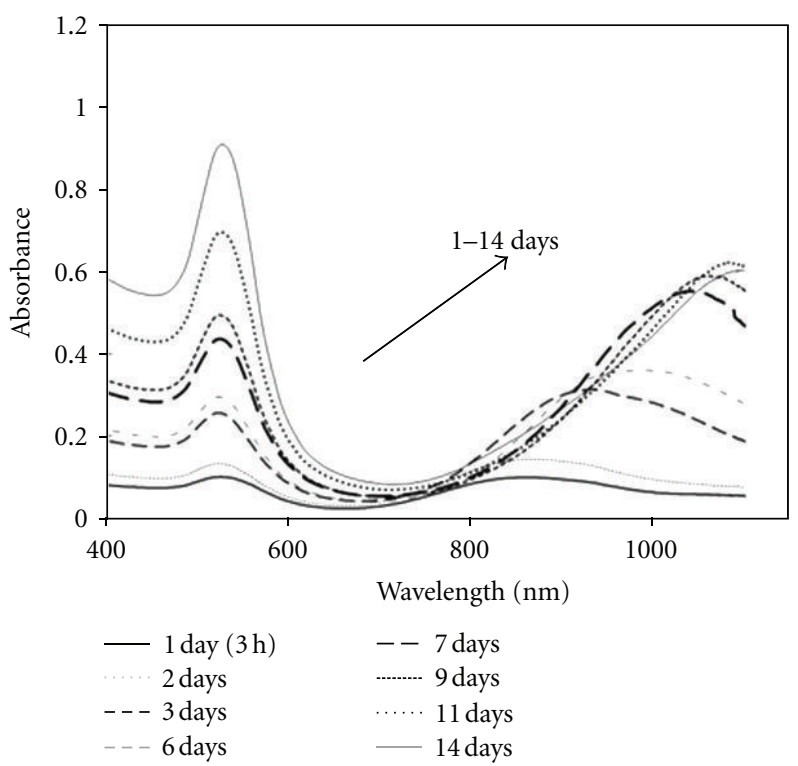

(c)

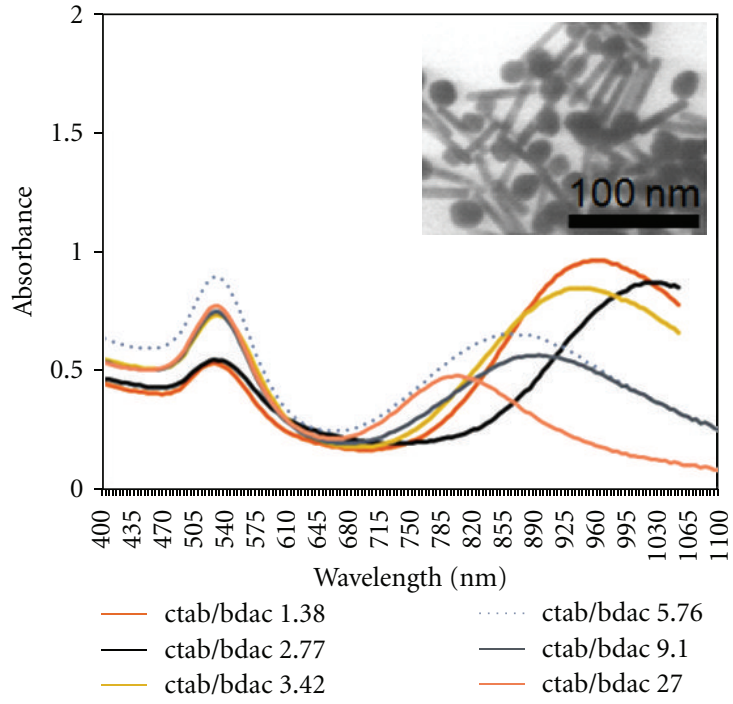

(b)

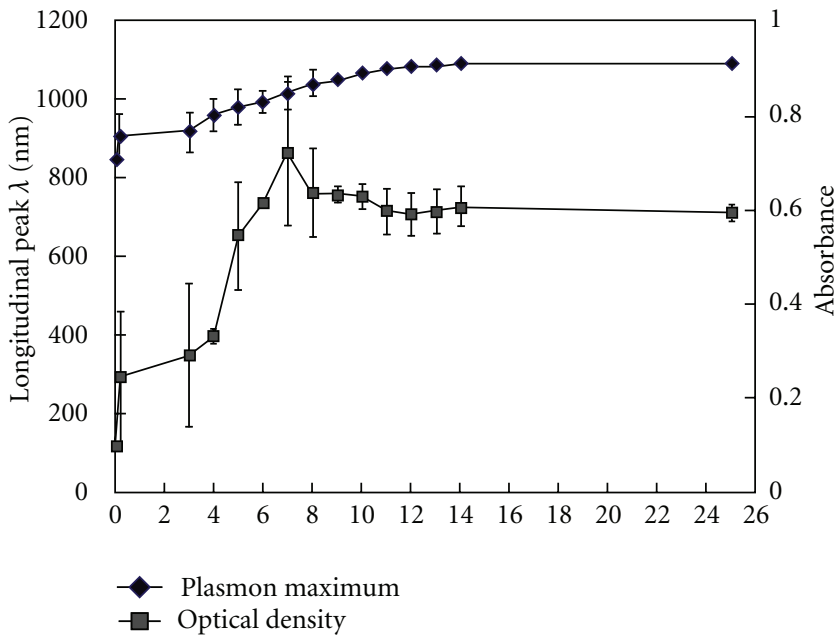

(d)

FIGURE 5: (a) Electron microscopy of long nanorods (AR: $8.9 \pm 0.6$ ) with plasmon bands $>850 \mathrm{~nm}$. (b) Effect of BDAC/CTAB ratio on the rod aspect ratios and absorption wavelength. High BDAC/CTAB ratio tends to form nanospheres in addition to long rods. (c) Temporal evolution of absorption spectrum over time demonstrating red shifts of the longitudinal bands to longer wavelength as the rod length increases. (d) Temporal evolution of increase in the optical density and longitudinal peak wavelength in the first 14 days and stop. The optical density increases over time indicating the multiply of rod numbers.

and seed will eventually cause a reduction in the aspect ratio and plasmonic peak, as shown above.

Nanoparticles of various shapes and sizes can be synthesized with different chemical functional groups. Therefore, to synthesize rod-shaped nanoparticles with absorption $>900 \mathrm{~nm}$, a binary surfactant system in growth solution was sought to enable production of higher aspect ratio during an extended aging period. Typically, a second surfactant $\mathrm{BDAC}$ is used in addition to CTAB at an appropriate molar ratio. Higher ratios of $\mathrm{BDAC/CTAB}$ tend to result in the formation of large amounts of spherical nanoparticles (Figure 5(b)). When the molar ratio of BDAC/CTAB to 2.77 was used in our study, the binary surfactant mixture was capable of fabricating long aspect ratio nanorods with plasmon bands over 1,000 nm (Figure 5(a): SEM; Figure 5(b): absorption spectrum). In contrast, using a growth solution containing BDAC showed little effect on the elongation as only nanospheres were produced. This result suggested that CTAB plays an important nanostructure-determining role in the rod growth under the influence of silver ions. 
Nanospheres usually start to grow upon addition of gold seeds to the bisurfactant growth solution. As shown in Figure 5(c), rod-shaped nanoparticles multiply within 3 hours, showing longitudinal plasmonic bands around $850-950 \mathrm{~nm}$. At this stage the aspect ratios of rods are approximately $5-5.5$. Since then, the growth process mainly contributes to the increase in the rod length. As the $\mathrm{Au}^{3+}$ ions are reduced to $\mathrm{Au}^{0}$, supply of gold atoms seems to be more favorable to join the ends of the nanocrystal than surfactant capped sides thereby elongating the rod length and resulting in a higher aspect ratio. It is known that the LSPR peak wavelength prolongs as the aspect ratio increases. As the aspect ratio increased over time, the plasmon peak gradually shifted to a longer wavelength in the NIR region during the first two weeks (Figure 5(d)). Afterwards, the growth process came to an end, and further incubation (14 25 days) failed to increase the length of the NRs. Because the growth solution has excess amount of surfactants, depletion of the gold supply from $\mathrm{Au}^{3+}$ ions in the solution is most likely to be the main reason for the stop.

Figure 5(d) shows a temporal evolution of long rods in absorbance spectrum over two weeks of growth and aging period. From the optical density, it shows that the production of rods (yield) accelerated during the first 7 days as the OD increased rapidly. Afterwards, a slight reduction in the OD was observed, and OD continued to remain steady over the 25 days. This indicates that the 8-10 days may be sufficient for a full growth of the long nanorods, and separation from the growth solution can be performed to harvest long rods with desired plasmonic wavelength. Considering the OD measurement is for the longitudinal band of rod-shaped nanoparticles, the slight dip in the optical density after 7 days suggests slowdown of the rod length elongation, rendering a relatively faster increase in the spherical formation as byproduct. In other words, a fraction of the rod becomes shorter and shorter in aspect ratio after depletion of the $\mathrm{Au}^{0}$ supply. It should be noted that although the data here only shows a peak around $1,050 \mathrm{~nm}$ due to the instrument limitation, rods of aspect ratio up to 10 with absorption band at $1,300 \mathrm{~nm}$ can be fabricated using the BDAC/CTAB method [24].

3.5. Effect of Centrifuge. After the growth of seed to rodshaped nanoparticles, centrifuge was an essential step for purification by separating excess chemical reactants and small sphere particles from rod-shaped nanoparticles. This process may affect the concentration, dispersion, and dimension of the rods. Therefore, the effect of the centrifuge speed and times on the longitudinal peak wavelength of the nanorods was investigated. A batch of $180 \mathrm{~mL}$ nanorods solution was divided to multiple samples, and, at baseline (before centrifuge), the GNRs showed a plasmon band at $772 \mathrm{~nm}$. The samples were then centrifuged at a speed of $5,500,7,000$, and $8,500 \mathrm{rpm}$, respectively, from one to three times. Readings of the absorption spectrum were taken at 4 , 8 , and 12 days after centrifuge to exam the stability of the purified nanorods. For all experimental conditions, the GNR samples showed a blue shift (reduction in the wavelength) of the peak after centrifugation. For example, for the samples centrifuged at 7,000 rpm, one time centrifuge resulted in a peak wavelength of $767 \mathrm{~nm}$, and two times caused a shift to $757 \mathrm{~nm}$, while centrifugation of three times resulted in a further shift down to $748 \mathrm{~nm}$. The magnitude of the peak wavelength shortening between one and two centrifuge times depends on the centrifuge speed (Figure 6). At 5,500 rpm, the shift was only $0.3 \%$ (from 768 to $766 \mathrm{~nm}$ ). The shift became $1.3 \%$ (from 767 to $757 \mathrm{~nm}$ ) at 7,000 rpm, while $8,500 \mathrm{rpm}$ centrifugation caused a $2.6 \%$ reduction from 768 to $749 \mathrm{~nm}$. Nevertheless, after three times of the centrifuge, the final plasmon band was stabilized with little variations for all tested speeds.

The centrifuge also played an important role in the stability of the GNRs as shown in their corresponding longitudinal peak wavelength (Figure 6). After one time centrifuge at $8,500 \mathrm{rpm}$, the plasmon band demonstrated a large variation during storage time from $768 \mathrm{~nm}$ (4 days) to $760 \mathrm{~nm}$ ( 8 days) and to $753 \mathrm{~nm}$ (12 days). The blue shift of the absorption band peak due to reduction in the aspect ratio over time suggested insufficient removal of the excess chemical reactants from the GNR sample; therefore, the aspect ratio of rods changed. This observation corroborated with other studies [24], and it is probably due to the increase in the width of the nanorods while the length is kept relatively unchanged in the remainder of growth solution after centrifuge. After two times of centrifuge, the change in the peak wavelength was less obvious at higher speed such as $8,500 \mathrm{rpm}$, and three times of centrifuge were sufficient to separate excess growth solution, and the GNR dimension remained unchanged over time. These results indicate that at lower centrifuge speed such as 5,500 to $7,000 \mathrm{rpm}$, three times of centrifuge may be necessary for purification. At $8,500 \mathrm{rpm}$ or higher, two-time centrifugation is most likely to be sufficient to produce stable and concentrated nanorod samples. It is suggested not to have more than 3 times of centrifugation based on our experiences.

\section{Conclusions}

We systematically studied the reaction parameters and centrifuge effect on the formation of rod-shaped gold nanoparticles in a relatively large quantity of $80-100 \mathrm{~mL}$. Although all chemicals are important in directing the nanostructure, silver ion concentration and seed/ $\mathrm{Au}^{3+}$ ratio were the most effective variations to adjust the absorption wavelength. This is consistent with the small quantity study reported by Murphy and El-Sayed groups. Since the molar concentrations of each ingredient for a large volume production multiply, even distribution by well mixing and precise control techniques play an essential role in success and determination of the final yield, uniformity, and stability. Temporal evolution of the gold nanorod dimensions fabricated by bisurfactant system was elucidated to follow the red shifts of longitudinal SPR peak wavelengths $>900 \mathrm{~nm}$ which is the limit for CTAB only method. In summary, nanorods of absorption bands from $600-1050 \mathrm{~nm}$ were fabricated with precise control of the AR from 1.5 to 8.9 in a relatively large quantity. 


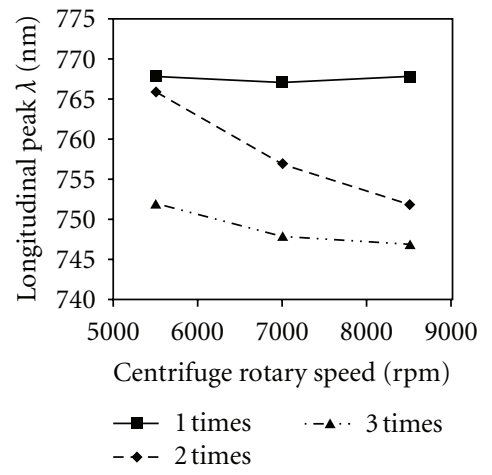

(a)
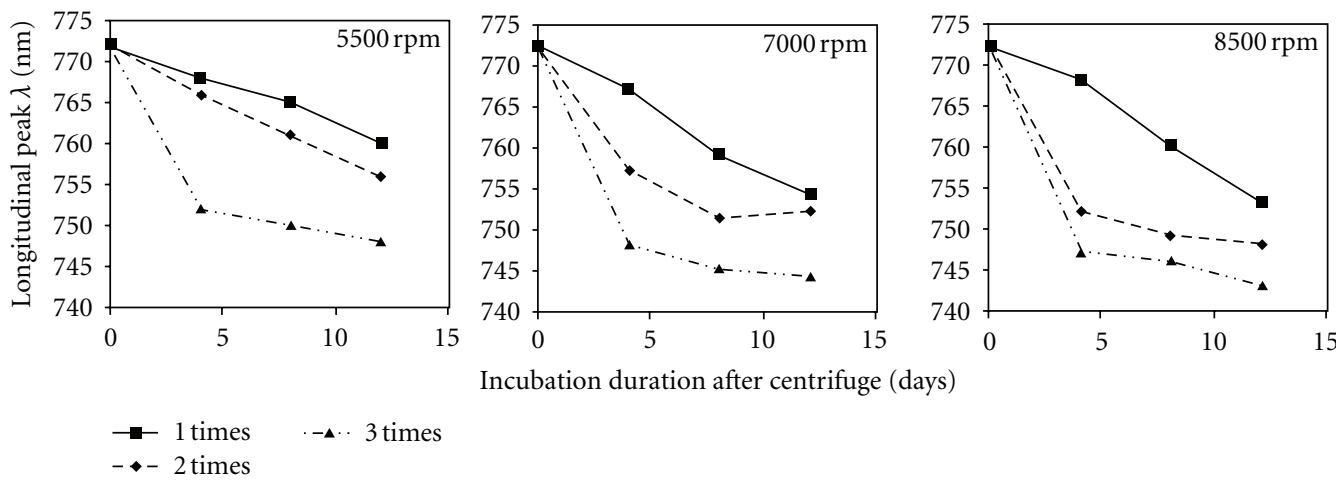

(b)

Figure 6: (a) Effects of centrifugal times and speed on the final purified nanorod optical properties. (b) Stability of the rods over 14 days depending on the centrifuge speed and times.

The mass production of these rod-shaped gold nanoparticles with controllable aspect ratio and tunable optical properties will provide a solid material foundation for biological and therapeutic applications.

\section{Acknowledgments}

This work was partly supported by San Antonio Area Foundation and UTSA CRSGP funds.

\section{References}

[1] J. Maier, "Nanoionics: ion transport and electrochemical storage in confined systems," Nature Materials, vol. 4, no. 11, pp. 805-815, 2005.

[2] Y. Namiki, T. Fuchigami, N. Tada et al., "Nanomedicine for cancer: lipid-based nanostructures for drug delivery and monitoring," Accounts of Chemical Research, vol. 44, no. 10, pp. 1080-1093, 2011.

[3] R. S. Sundaram, M. Steiner, H.-Y. Chiu et al., "The graphenegold interface and its implications for nanoelectronics," Nano Letters, vol. 11, no. 9, pp. 3833-3837, 2011.

[4] A. Bhirde, J. Xie, M. Swierczewska, and X. Chen, "Nanoparticles for cell labeling," Nanoscale, vol. 3, no. 1, pp. 142-153, 2011.

[5] R. A. Frimpong and J. Z. Hilt, "Magnetic nanoparticles in biomedicine: synthesis, functionalization and applications," Nanomedicine, vol. 5, no. 9, pp. 1401-1414, 2010.
[6] L. Dykman and N. Khlebtsov, "Gold nanoparticles in biomedical applications: recent advances and perspectives," Chemical Society Reviews, vol. 41, no. 6, pp. 2256-2282, 2012.

[7] D. A. Giljohann, D. S. Seferos, W. L. Daniel, M. D. Massich, P. C. Patel, and C. A. Mirkin, "Gold nanoparticles for biology and medicine," Angewandte Chemie, vol. 49, no. 19, pp. 32803294, 2010.

[8] E. C. Dreaden, A. M. Alkilany, X. Huang, C. J. Murphy, and M. A. El-Sayed, "The golden age: gold nanoparticles for biomedicine," Chemical Society Reviews, vol. 41, no. 7, pp. 2740-2779, 2012.

[9] J. L. West and N. J. Halas, "Engineered nanomaterials for biophotonics applications: improving sensing, imaging, and therapeutics," Annual Review of Biomedical Engineering, vol. 5, pp. 285-292, 2003.

[10] S. E. Skrabalak, L. Au, X. Li, and Y. Xia, "Facile synthesis of Ag nanocubes and Au nanocages," Nature Protocols, vol. 2, no. 9, pp. 2182-2190, 2007.

[11] J. Zhang, M. R. Langille, M. L. Personick, K. Zhang, S. Li, and C. A. Mirkin, "Concave cubic gold nanocrystals with highindex facets," Journal of the American Chemical Society, vol. 132, no. 40, pp. 14012-14014, 2010.

[12] J. E. Millstone, S. Park, K. L. Shuford, L. Qin, G. C. Schatz, and C. A. Mirkin, "Observation of a quadrupole plasmon mode for a colloidal solution of gold nanoprisms," Journal of the American Chemical Society, vol. 127, no. 15, pp. 5312-5313, 2005.

[13] E. Petryayeva and U. J. Krull, "Localized surface plasmon resonance: nanostructures, bioassays and biosensing-a review," Analytica Chimica Acta, vol. 706, no. 1, pp. 8-24, 2011. 
[14] S. M. Marinakos, S. Chen, and A. Chilkoti, "Plasmonic detection of a model analyte in serum by a gold nanorod sensor," Analytical Chemistry, vol. 79, no. 14, pp. 5278-5283, 2007.

[15] T. A. Taton, C. A. Mirkin, and R. L. Letsinger, "Scanometric DNA array detection with nanoparticle probes," Science, vol. 289, no. 5485, pp. 1757-1760, 2000.

[16] L. R. Hirsch, J. B. Jackson, A. Lee, N. J. Halas, and J. L. West, "A whole blood immunoassay using gold nanoshells," Analytical Chemistry, vol. 75, no. 10, pp. 2377-2381, 2003.

[17] H. Huang, C. He, Y. Zeng et al., "A novel label-free multithroughput optical biosensor based on localized surface plasmon resonance," Biosensors and Bioelectronics, vol. 24, no. 7, pp. 2255-2259, 2009.

[18] C. Yu and J. Irudayaraj, "Multiplex biosensor using gold nanorods," Analytical Chemistry, vol. 79, no. 2, pp. 572-579, 2007.

[19] K. S. Lee and M. A. El-Sayed, "Gold and silver nanoparticles in sensing and imaging: sensitivity of plasmon response to size, shape, and metal composition," Journal of Physical Chemistry $B$, vol. 110, no. 39, pp. 19220-19225, 2006.

[20] P. K. Jain, S. Eustis, and M. A. El-Sayed, "Plasmon coupling in nanorod assemblies: optical absorption, discrete dipole approximation simulation, and exciton-coupling model," Journal of Physical Chemistry B, vol. 110, no. 37, pp. 1824318253, 2006.

[21] C. Yu and J. Irudayaraj, "Quantitative evaluation of sensitivity and selectivity of multiplex nanoSPR biosensor assays," Biophysical Journal, vol. 93, no. 10, pp. 3684-3692, 2007.

[22] T. K. Sau and C. J. Murphy, "Seeded high yield synthesis of short Au nanorods in aqueous solution," Langmuir, vol. 20, no. 15, pp. 6414-6420, 2004.

[23] C. J. Murphy, T. K. Sau, A. M. Gole et al., "Anisotropic metal nanoparticles: synthesis, assembly, and optical applications," Journal of Physical Chemistry B, vol. 109, no. 29, pp. 1385713870, 2005.

[24] B. Nikoobakht and M. A. El-Sayed, "Preparation and growth mechanism of gold nanorods (NRs) using seed-mediated growth method," Chemistry of Materials, vol. 15, no. 10, pp. 1957-1962, 2003.

[25] K. L. Kelly, E. Coronado, L. L. Zhao, and G. C. Schatz, "The optical properties of metal nanoparticles: the influence of size, shape, and dielectric environment," Journal of Physical Chemistry B, vol. 107, no. 3, pp. 668-677, 2003. 

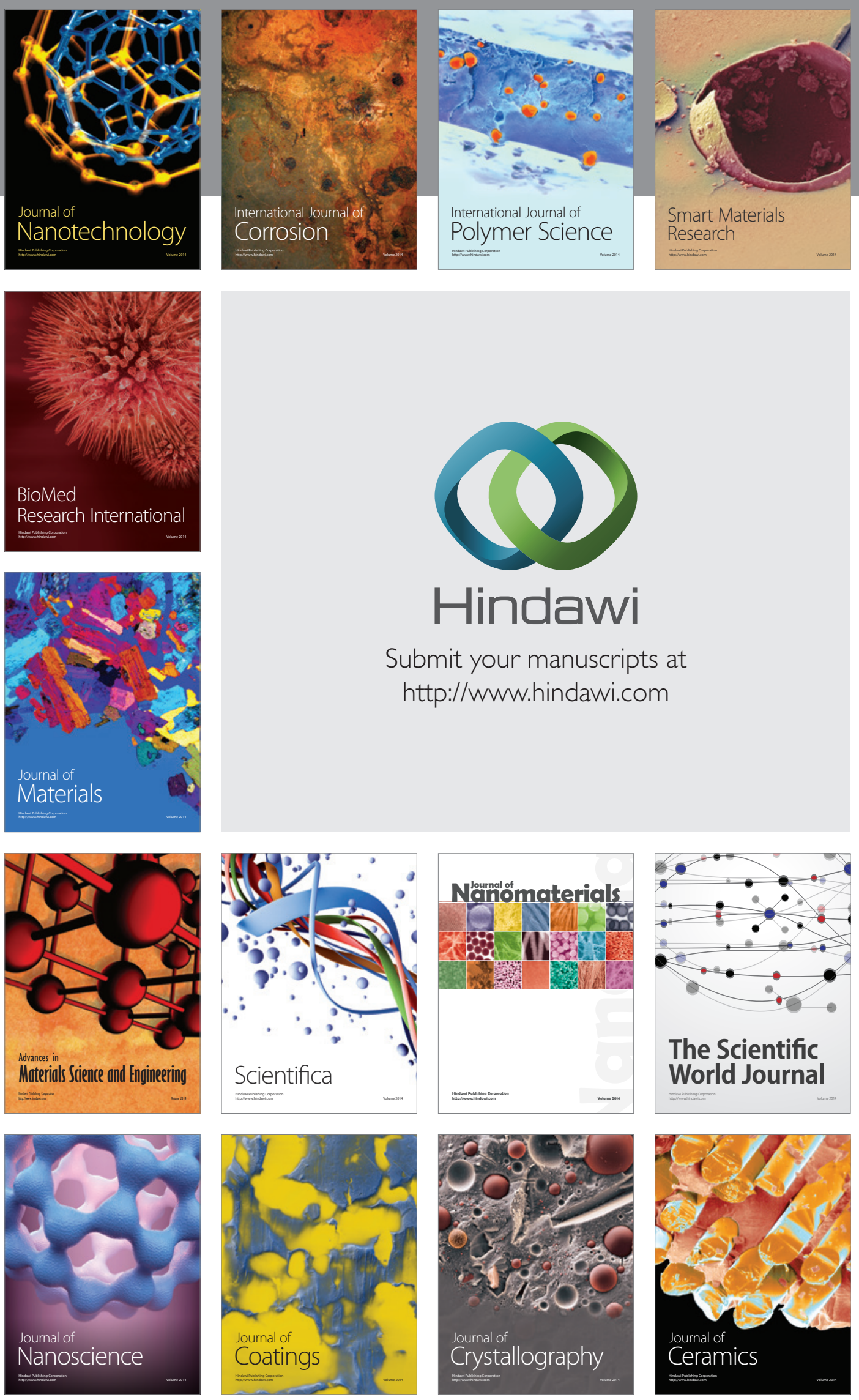

The Scientific World Journal

Submit your manuscripts at

http://www.hindawi.com

\section{World Journal}

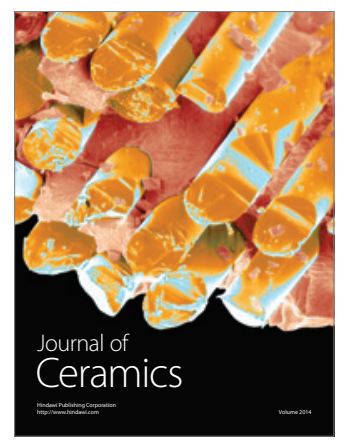

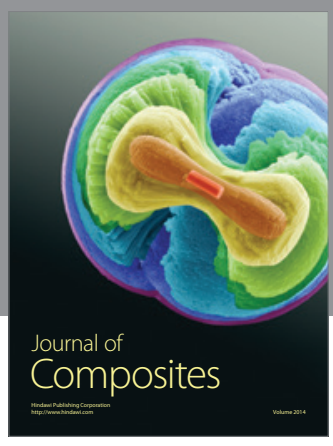
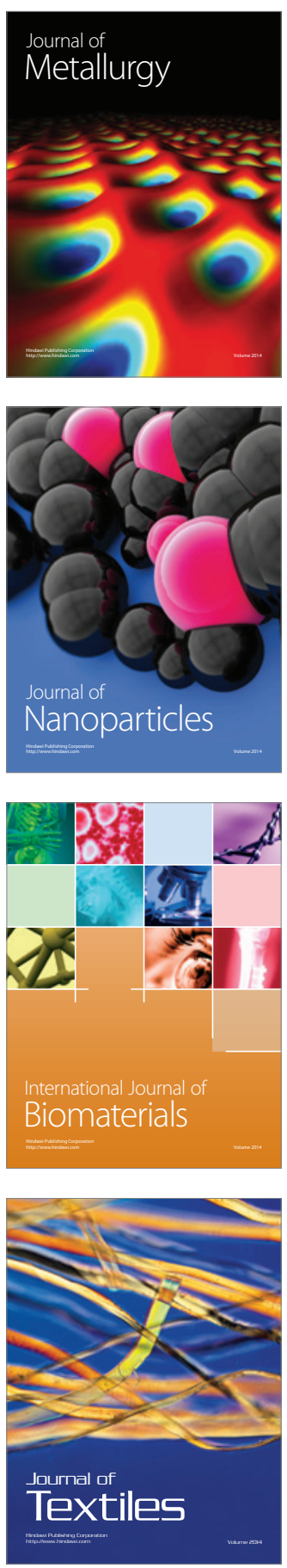